phase within them. Inside the epicentral region (later data recorded at stations on opposite sides of the region), the pre-1967 crack density was slightly higher than outside but again the cracks were mostly saturated. And again the $V_{\mathrm{P}} / V_{\mathrm{s}}$ decrease was accompanied by a decrease, albeit rather more marked, in crack density. But thereafter the picture changed. The subsequent increase in $V_{\mathrm{P}} / V_{\mathrm{S}}$ was again related to resaturation but was now accompanied by an increase in crack density.

In summary, then, there are significant differences in the precursory processes inside and outside the immediate epicentral zone. Close to the main shock area the sequence is dilatancyresaturation-dilatancy, whereas further away the sequence becomes dilatancyresaturation-relaxation.

Extensive cracking is common to both regions, however, and extends over a much wider zone than some workers have previously supposed. And again contrary to some previous views, the observed decrease in $V_{\mathrm{P}} / V_{\mathrm{S}}$ is not due to the formation of new cracks but to a change from saturated to dry cracks. Indeed, changes in the saturation state of the cracks are apparently more important on the whole than changes in crack numbers.

\section{Opening up the Universe}

\section{from $P . C . W$. Davies}

Most cosmologists now accept that the Universe began with a bang. But how will it end? The question has been of long-standing interest to both theologians and scientists, though the former have enjoyed greater success in producing answers. In recent months, however, evidence has been accumulating from diverse astronomical sources which consistently points toward a definite scenario for the future of the Universe. Although there is no question of a unanimous verdict at this stage, a movement of opinion among the pundits is becoming perceptible.

All discussion of this matter takes place within the context of the standard model for the Universe. In this standard model, the Universe moves in compliance with Einstein's general theory of relativity. This motion is visible to us as a general pattern of expansion, and because of the apparent large-scale homogeneity and isotropy, this expansion is assumed to be everywhere uniform.

If Einstein's equations are solved for such a uniform model universe they yield a two-parameter family of motions. All of these solutions predict that the expansion began a few billion years ago, when the Universe was in a very dense condition. The onset of the expansion, expected to be very hot, is the big bang. The past history of the Universe is therefore, in broad outline, fairly unambiguous according to this theory.

As regards the future motion of the cosmos, all the solutions predict a gradual decrease in the expansion rate. Where they differ is in whether the decrease is strong enough to arrest the expansion and bring about recontraction, with the Universe falling back on itself to end up in a bang much like the one from which it originated. The alternative is for the expansion to continue for ever, with the Universe slowly sinking into thermodynamic equilibrium, after which little of significance will occur.

In principle it is easy to decide between these alternatives. Observations of the rate of recession of distant galaxies, seen as they were in the remote past, should indicate how the expansion rate has slowed since then. Alternatively, measurements of the present energy density in the Universe enables the gravitating effect to be calenable the gravitating effect to be calanswer (through the general theory of relativity) as to how vigorously this gravitation is slowing the Universe down.

In practice, both types of observation are difficult to perform and complicated by many contentious sideissues. Now a paper has appeared in the Astrophysical Journal (194, 543; 1975) by Gott and Gunn from Caltech and Schramm and Tinsley from the University of Texas in which many of the observations and their theoretical ramifications are examined in detail. Gott et al. opt for an ever-expanding (or open) universe. Some of the arguments they use were presented by Gunn at the Seventh Texas Symposium on Relativistic Astrophysics (see the report from John Faulkner, Nature, 253, 231; 1975).

The authors parameterise their models in terms of the Hubble parameter $H_{0}$ (expansion rate at present epoch) and $\Omega$, the ratio of the observed density of energy to the critical density required to collapse the Universe. For an ever expanding Universe, $\Omega \leqslant 1$. Their figure 1 shows the constraints obtained in their paper on these parameters.

Although $H_{0}$ may be measured directly $\left(30<H_{0}<120 \mathrm{~km} \mathrm{~s}^{-1} \mathrm{Mpc}^{-1}\right)$ the age of the Universe, $t_{1}$ ( 8 to 18 billion years), is a more severe constraint. A direct estimation of the deceleration is complicated by evolutionary effects in both galaxies and QSOs, which tend to result in an overestimation of the deceleration parameter, $q_{0}(=\Omega / 2)$. To play safe an upper limit of 2 is placed on this parameter.
In contrast, the density $(\Omega)$ measurements tend to be underestimates, first because we only see the luminous matter in the Universe (stars, gas) and second because energy density may reside in the form of very low energy radiation (gravitons, neutrinos). Various methods for estimating $\Omega$ are critically described in the paper. Three independent estimates of the relative density of galaxies alone (denoted $\Omega^{*}$ ) are used to obtain a value $0.05 \pm 0.01$, and various arguments reviewed as to why any intergalactic matter would not be sufficient to give $\Omega>1$.

In addition, a mention is made of recent theoretical work on the production of deuterium by nucleosynthesis in the hot big bang. The fraction of deuterium produced $(\mathrm{D} / \mathrm{H}$ or ratio of deuterium to hydrogen) turns out to be very sensitive to the present energy density of the Universe. Using the results of measurements of galactic deuterium abundance, it is concluded that remarkably narrow ranges of $\Omega$ and $H_{0}$ are permitted in which $0.05<\Omega<0.09$ and $49<H_{n}<65 \mathrm{~km} \mathrm{~s}^{-1} \mathrm{Mpc}^{-1}$, predicting an ever-expanding Universe by a wide margin. The possibility of galactic deuterium production and nonstandard big bang physics is briefly reviewed.

The authors conclude that the density of the Universe is low, $\Omega \sim 0.06 \pm 0.02$, and a recontraction is ruled out. The most persuasive part of their argument is the fact that an ever-expanding Universe follows consistently from all the different sources of data, whereas to produce a recontracting model, a number of ad hoc assumptions are necessary. It is always possible to invoke exotic processes in the big bang, or to conjecture that most of the mass of the Universe is in the form of undetectable gravitational waves or black holes. But such conjectures are extremely hard to falsify with current technology, and seem somewhat contrived.

If Gott et al. are right, then instead of the Universe going out in a blaze of glory by recontraction, collapse and final cremation, it is doomed to everlasting frozen stagnation. when the stars go out in a few dozen billion years.

\section{Erratum}

IN the article "Chilling Statistics on Cyprus" by Peter J. Smith (Nature, $253,500 ; 1975)$ magnetic vectors are mentioned in the last sentence of the fourth paragraph. This is incorrect and the sentence should read: "In other words, on each side of the intrusion zone the chilled margins will all lie in the same direction (they will all be 'one way') and the degree of 'one way chilling' will be $100 \%$." 